\title{
Bromodomain PHD-finger transcription factor promotes glioma progression and indicates poor prognosis
}

\author{
YANLING PAN ${ }^{1,2}$, FENG YUAN ${ }^{2}$, YIJIE LI $^{2}$, GUOPING WANG ${ }^{2}$, ZHIREN LIN $^{2}$ and LONGHUA CHEN ${ }^{1}$ \\ ${ }^{1}$ Department of Radiation Oncology, Nanfang Hospital, Southern Medical University, Guangzhou, Guangdong 510515; \\ ${ }^{2}$ Department of Radiation Oncology, Haikou People's Hospital, Haikou, Hainan 570208, P.R. China
}

Received June 25, 2018; Accepted October 19, 2018

DOI: $10.3892 /$ or.2018.6832

\begin{abstract}
Glioma is one of the most deadly central nervous system tumors around the world. Uncontrollable cell proliferation and invasion are key factors of cancer progression as well as glioma. Available evidence suggests that bromodomain PHD-finger transcription factor (BPTF) plays an important role in stem cell proliferation and differentiation, as well as in progression of some tumors, but there is little data on glioma. Therefore, the present study aimed to explore the functional role and potential clinical value of BPTF in glioma. Public database, real-time PCR and western blotting were used to detect the expression of BPTF in glioma tissue and cells. The relationship between BPTF with clinicopathological features and the prognosis of glioma patients was analyzed by immunohistochemical staining in 113 cases of paraffin-embedded primary glioma specimens. Furthermore, cytological experiments were conducted to elucidate the functional role of BPTF in glioma U251 cells, as well as the potential molecular mechanism. The expression of BPTF in glioma tissues was significantly higher than that in normal brain tissues. The association analysis results revealed that high BPTF expression was significantly associated with WHO grade and tumor size. Survival analysis revealed that the BPTF high-expression group had poorer overall survival (OS) and progression-free survival (PFS) compared with the low-expression group. Univariate and multivariate Cox regression analyses revealed that BPTF expression was an independent prognostic factor for the OS and PFS of glioma patients. Cytological experiments revealed that BPTF overexpression could significantly promote the proliferation, migration and invasion of human glioma U251 cells. A study of the underlying mechanism
\end{abstract}

Correspondence to: Dr Longhua Chen, Department of Radiation Oncology, Nanfang Hospital, Southern Medical University, 1838 North Guangzhou Avenue, Guangzhou, Guangdong 510515, P.R. China

E-mail: chenlonghuasci@163.com

Key words: glioma, bromodomain PHD finger transcription factor, prognosis, progression, MYC indicated that BPTF promoted glioma progression via MYC signaling. Our results preliminarily indicated that BPTF promoted glioma progression via MYC signaling and may be a potential prognostic biomarker and therapeutic target for glioma patients.

\section{Introduction}

Tumors of the central nervous system (CNS) represent a relatively rare but serious health burden in terms of morbidity and mortality (1). Approximately $2 \%$ of all adult malignancies are brain tumors, and $80 \%$ of them are glioma (2). Despite important progress in diagnostic and therapeutic techniques over the past decades, the prognosis for patients with high-grade gliomas (WHO grade III and IV tumors) remains discouraging (3). One of the most important reasons for this, is that the molecular mechanism of glioma progression is still unclear. Thus, it is essential to understand the mechanism of glioma invasion and metastasis and develop new therapeutic strategies.

It is already known that the main differences between tumor cells and normal cells lie in the different cell properties. Hanahan and Weinberg have concluded these properties as the ten hallmarks of cancer, such as sustaining proliferative signaling, activating invasion and metastasis (4). However, the precise mechanisms of regulating these hallmarks still need to be clarified. Existing researches have demonstrated that signaling pathways such as MYC, TGF- $\beta /$ Smad and Notch are involved in the occurrence and development of cancer $(5,6)$. Thereby, the malignant biological behaviors of cancer cells which differentiate from normal cells are mainly a result of the aberrancy of these signaling pathways.

Bromodomain PHD finger transcription factor (BPTF) is located at chromosome 17q24.3 and is involved in transcriptional regulation and chromatin remodeling in many biological processes (7). The completed sequence of human BPTF encodes a predicted protein of 2,781 amino acids, which contain typical features of bromodomain, two PHD fingers and an extensive glutamine-rich acidic domain (8). Previous research indicated that BPTF mainly participated in embryonic development (9). The multiple signaling mechanisms in embryonic development are always associated with tumor development and progression. Another study revealed that BPTF could regulate chromatin remodeling and control 
neuroectodermal differentiation, which was also correlated with cancer development (10). In addition, BPTF was also revealed to be involved in a variety of tumor-related signaling pathways such as MYC and Smad $(10,11)$. Furthermore, previous studies confirmed the cancer-promoting role of BPTF in colorectal and lung cancer $(12,13)$. These findings indicated that BPTF may play an important oncogenic role in cancer progression. However, there are no studies on the functional role and molecular mechanisms of BPTF in glioma.

In the present study, we first examined the expression of BPTF in glioma and assessed the relationship of BPTF expression with the prognosis of glioma patients. We further identified the functional role of BPTF in the U251 cell line and explored the potential molecular mechanism. Therefore, our findings provided a new understanding of BPTF in glioma progression and may reveal a novel potential therapeutic target for glioma.

\section{Materials and methods}

Patients and tissue specimens. Ten pairs of fresh-frozen glioma tissue and their corresponding normal brain tissue were randomly selected. Another 113 cases of paraffin-embedded glioma tissue samples were also randomly obtained from Haikou People's Hospital from January 2010 to December 2014. All the gliomas were pathologically confirmed by 2 independent pathologists according to the 2007 WHO classification. The fresh-frozen glioma tissue and normal brain tissue were analyzed by real-time quantitative reverse-transcription polymerase chain reaction (real-time PCR) and western blotting (WB). The paraffin-embedded glioma tissue samples were detected by immunohistochemical staining (IHC). All patients eligible for the present study had undergone surgery, and were routinely followed-up and detailed clinicopathological and survival data was collected. Among them, 52 were female and 61 were male and the median age was 47 years (range, 21-82 years). Magnetic resonance imaging (MRI) or contrast-enhanced MRI was performed every 6 months to detect tumor relapse or metastasis after surgery. Overall survival (OS) was defined as the time from the surgery to the death of the patient or the last follow-up visit. Progression-free survival (PFS) was defined as the time from the surgery to the first evidence of recurrence, progression, or death. All patients provided signed written informed consent. The study was approved by the Ethics Committee of Haikou People's Hospital in accordance with the Declaration of Helsinki.

Real-time PCR. Total RNA was extracted from the fresh-frozen specimens using TRIzol reagent (Invitrogen; Thermo Fisher Scientific, Inc., Waltham, MA, USA) according to the specifications of the instructions. Real-time PCR was performed using the SYBR-Green Real-Time PCR Master Mix (Toyobo Co., Ltd., Osaka, Japan) as the manufacturer's instructions described. An ABI 7100 Real-Time Quantitative PCR System (Applied Biosystems, Foster City, CA, USA) was used, in which each reaction $(25 \mu \mathrm{l})$ contained $10 \mu \mathrm{l}$ PCR Master Mix (Ambion; Thermo Fisher Scientific, Inc.) and $1.3 \mu \mathrm{l}$ RT product, and each sample was analyzed in triplicate. The PCR reaction was conducted at $95^{\circ} \mathrm{C}$ for $10 \mathrm{~min}$, followed by 40 cycles at $95^{\circ} \mathrm{C}$ for $15 \mathrm{sec}$ and $60^{\circ} \mathrm{C}$ for $1 \mathrm{~min}$. The reactions were performed in two independent assays. The primers of BPTF were as follows: Forward, 5'-GGAGAGATGTTGGTCCTTATGGC-3' and reverse, 5'-CTTTCCTCTGAGGTGTAGGCGT-3'; $\beta$-actin was used as a control using the following primers: Forward, 5'-CAC CATTGGCAATGAGCGGTTC-3' and reverse, 5'-AGGTCT TTGCGGATGTCCACGT-3'. The results were analyzed using the $2^{-\Delta \Delta \mathrm{Cq}}$ method as previously described (14).

Western blot (WB) analysis. Total proteins of fresh-frozen glioma and normal brain tissues were extracted using RIPA lysis buffer (Thermo Fisher Scientific, Inc.) and separated by $10 \%$ sodium dodecyl sulfate-polyacrylamide gel electrophoresis (SDS-PAGE) then transferred onto polyvinylidene fluoride (PVDF) membranes (EMD Millipore, Billerica, MA, USA) as previously described (15). The protein concentration was detected by bicinchoninic acid (BCA) protein assay (Pierce Chemical, Rockford, IL, USA) according to the manufacturer's instructions. The blotted membranes were incubated with antihuman BPTF antibody (band size, $324 \mathrm{kDa}$; dilution 1:1,000; cat. no. ab72036; Abcam, Cambridge, MA, USA), or C-MYC antibody (band size, $67 \mathrm{kDa}$; dilution 1:1,000; cat. no. sc-47694; Santa Cruz Biotechnology, Santa Cruz CA, USA) or $\beta$-actin antibody (band size, $42 \mathrm{kDa}$; dilution 1:2,000; cat. no. ab173838; Abcam) at $4^{\circ} \mathrm{C}$ overnight, then incubated with an appropriate secondary antibody (dilution 1:2,000; cat. no. sc-2004; dilution 1:3,000; cat. no. sc-2005; Santa Cruz Biotechnology) for $30 \mathrm{~min}$ at room temperature. The western blotting band was detected by enhanced chemiluminescence reagents (Thermo Fisher Scientific, Inc.) and the band density was assessed by ImageJ software (version k1.45 for windows; National Institutes of Health, Bethesda, MD, USA) and the density detection was repeated three times.

Immunohistochemistry (IHC). The tissue specimens were fixed with $10 \%$ formalin and then embedded in paraffin; 4-mm sections were cut and placed on silane-coated slides for immunohistochemical analysis. Each slide of the specimens was stained with hematoxylin and eosin (H\&E) and microscopically examined to confirm the pathological diagnosis. The paraffin-embedded sections were dewaxed and pretreated with $0.01 \mathrm{M}$ sodium citrate buffer $(\mathrm{pH} 6.0)$ for $15 \mathrm{~min}$ at $95^{\circ} \mathrm{C}$ for tissue antigen retrieval. Then, these sections were incubated with $3 \%$ hydrogen peroxide for $20 \mathrm{~min}$ at room temperature to block endogenous peroxidase. After rinsing three times for $2 \mathrm{~min}$ with phosphate-buffered saline (PBS), 10\% goat serum was added as a blocking liquid, and incubated for $15 \mathrm{~min}$ at room temperature. Next, the serum was removed, and the appropriate diluted primary antibody (BPTF; dilution 1:250; cat. no. ab72036; Abcam) was added to each section and incubated overnight at $4^{\circ} \mathrm{C}$. After rinsing the primary antibody using PBS, the sections were handled according to the manufacturer's recommendations (PV-9000; Beijing Zhongshan Golden Bridge Biotechnology Co., Ltd., Beijing, China) and counterstained with hematoxylin; then dehydration was conducted by graded ethanol followed by the addition of xylene to render the sections transparent. Finally, the slides were covered with neutral balsam, and then observed and mounted with a Leica DM2000 optical microscope 
(Leica Microsystems, Wetzlar, Germany) and ImageJ k1.45 software (National Institutes of Health). The staining intensity of BPTF was scored according to a previous study: - (negative), + (weak), ++ (moderate) and +++ (strong). Stained tissues with ' - ' and ' + ' were considered as the BPTF low-expression group, and ' ++ ' and ' +++ ' were considered as the BPTF high-expression group (13). The staining was evaluated by two separated senior pathologists on a multi-head microscope with anonymous patient information.

Lentiviral vector construction and cell transduction. Full-length human BPTF overexpression clone plasmid and targeting shRNA (shRNA) lentivirus and their control vectors were purchased from Shanghai GeneChem Co., Ltd. (Shanghai, China). The transfection process was conducted according to the manufacturer's instructions. Puromycin $(2 \mu \mathrm{g} / \mathrm{ml})$ (Thermo Fisher Scientific, Inc.) was used to select stable clones if necessary. The transfection efficiency was assessed by fluorescence microscopy, real-time PCR and western blotting.

Proliferation assay. The proliferation capacity of cancer cells was assessed by colony formation assay. Cells were seeded into 6-well plates with a density of $5 \times 10^{2}$ cells/well, and cultured for $\sim 2$ weeks with fresh medium replaced every week. Then colonies were stained with crystal violet and counted by ImageJ software k1.45 (National Institutes of Health) and only the colonies containing $>50$ cells were counted and plotted. All the experiments were replicated in triplicate.

Migration and invasion assays. Migration ability of cancer cells was assessed by wound-healing and Transwell assays. In the wound-healing assay, the cells were cultured into 6-well plates at a density of $2 \times 10^{5}$ cells/well. When the cells grew to $90 \%$ confluence, they were incubated with mitomycin-C $(10 \mu \mathrm{g} / \mathrm{ml})$ for $1 \mathrm{~h}$ to suppress proliferation and then starved in serum-free medium for $24 \mathrm{~h}$. An artificial wound was created by scraping the cell confluent monolayer with a $10-\mu 1$ pipette tip. The migration gap of the wound was assessed after $48 \mathrm{~h}$. The migration and invasion potential was also evaluated by Transwell assay. The cells were treated with mitomycin-C $(10 \mu \mathrm{g} / \mathrm{ml})$ for $1 \mathrm{~h}$ at $37^{\circ} \mathrm{C}$ in serum-free medium and then plated into the upper chamber. For the migration assay the cells were seeded with a density of $2 \times 10^{4}$ cells/insert, and for the invasion assay cells are seeded into the top chamber coated with Matrigel (BD Biosciences, Franklin Lakes, NJ, USA) at a density of $4 \times 10^{4}$ cells/insert. Then DMEM with $10 \%$ fetal bovine serum (FBS) was added into the bottom chamber. After incubation for 24-48 h, the cells adhering to the lower membrane of the inserts were stained with $0.1 \%$ crystal violet and observed by a Leica DM2000 optical microscope (Leica Microsystems) then counted by ImageJ k1.45 software (National Institutes of Health). All the experiments were replicated in triplicate.

Public database analysis. The public database Oncomine (https://www.oncomine.org) was used for analyzing the expression of BPTF in glioma. 'BPTF' was used as a keyword in the Oncomine search, 'Cancer vs. Normal Analysis' was chosen as the primary filter, and 'brain and CNS cancer' was chosen as the cancer type, and 'mRNA' was chosen as the data type. The expression of BPTF was presented in multiple data sets including Rickman's and Murat's. The BPTF expression level was log-transformed and median-centered per array for analysis. The GCBI (http://www.gcbi.com.cn) and COXPRESdb (http://coxpresdb.jp) databases were used to analyze the interaction and co-expressed genes with BPTF.

Statistical analysis. All data were analyzed using the SPSS statistical software, version 18.0, for Windows (SPSS, Inc., Chicago, IL, USA). A Student's t-test (two-tailed) was used for statistical analysis of continuous data between two groups such as the relative expression level of BPTF in tumor and normal brain tissue. Multiple comparisons of continuous data among the control, BPTF-overexpression and BPTF-knockdown groups was analyzed by one-way ANOVA test followed by Tukey's post hoc test. Pearson Chi-square test was used for all of the categorical data. Survival curves were constructed using the Kaplan-Meier method and evaluated using the log-rank test. The univariate and multivariate Cox proportional hazards regression models were established to identify factors that were related or independently associated with the survival of glioma patients. A P-value of $<0.05$ was considered to indicate a statistically significant difference.

\section{Results}

BPTF expression is significantly elevated in gliomas tissue. We first analyzed BPTF expression by utilizing the public database Oncomine. The results revealed that the expression level of BPTF in brain tumor tissue was significantly higher than that in normal brain tissue in most of the public data (Fig. 1A). Since astrocytoma and glioblastoma account for $>70 \%$ of total glioma cases, we then chose Rickman's (astrocytoma) and Murat's (glioblastoma) data for further analysis. The results revealed that BPTF expression in astrocytoma and glioblastoma were both higher than that in normal brain tissue (Fig. 1B and C). Then, the BPTF mRNA expression of 8 pairs of fresh-frozen glioma tumor tissue and corresponding normal brain tissue from our hospital were also detected by real-time PCR. The results revealed that glioma tissues had significantly higher BPTF mRNA expression levels than the corresponding normal brain tissues (Fig. 1D). Consistent with the mRNA expression, the western blotting results also revealed that the expression of BPTF protein in glioma tissue was significantly higher than that in the corresponding normal brain tissue (Fig. 1E). These data clearly demonstrated that BPTF expression was significantly elevated in gliomas tissues.

High BPTF expression predicts poor prognosis in glioma patients. To further explore the BPTF expression pattern in glioma clinical samples and the correlation with clinicopathological characteristics and survival, we detected the BPTF protein expression in 113 cases of paraffin-embedded glioma tissues by immunohistochemistry. Immunohistochemical staining revealed BPTF positive staining mainly expressed in the cytoplasm and/or the nuclei of the tumor cells (Fig. 2A). There were $69(69 / 113,61.1 \%)$ cases that had BPTF positive staining, including $46(46 / 113,40.7 \%)$ cases of high BPTF expression. Then, we analyzed the expression of BPTF with clinicopathological features, and found that high 
Table I. BPTF expression and clinicopathological features of 113 glioma cases.

\begin{tabular}{|c|c|c|c|c|}
\hline \multirow[b]{2}{*}{ Variables } & \multirow[b]{2}{*}{ Total N (113) } & \multicolumn{2}{|c|}{ BPTF expression } & \multirow[b]{2}{*}{ P-value } \\
\hline & & Low (67) & High (46) & \\
\hline $\operatorname{Sex}$ & & & & 0.482 \\
\hline Female & 52 & 29 & 23 & \\
\hline Male & 61 & 38 & 23 & \\
\hline Age (years) & & & & 0.407 \\
\hline$\leq 50$ & 66 & 37 & 29 & \\
\hline$>50$ & 47 & 30 & 17 & \\
\hline Location & & & & 0.478 \\
\hline Frontal & 25 & 13 & 12 & \\
\hline Temporal & 34 & 23 & 11 & \\
\hline Parietal & 18 & 8 & 10 & \\
\hline Occipital & 17 & 11 & 6 & \\
\hline Others & 19 & 12 & 7 & \\
\hline Tumor size $(\mathrm{cm})$ & & & & $<0.001$ \\
\hline$\leq 5$ & 64 & 48 & 16 & \\
\hline$>5$ & 49 & 19 & 30 & \\
\hline Cystic change & & & & 0.335 \\
\hline Absence & 77 & 48 & 29 & \\
\hline Presence & 36 & 19 & 17 & \\
\hline Necrosis & & & & 0.554 \\
\hline Absence & 81 & 50 & 32 & \\
\hline Presence & 32 & 17 & 14 & \\
\hline WHO grade & & & & 0.001 \\
\hline I and II & 66 & 48 & 18 & \\
\hline III and IV & 47 & 19 & 28 & \\
\hline KPS score & & & & 0.288 \\
\hline$\leq 90$ & 51 & 33 & 18 & \\
\hline$>90$ & 62 & 34 & 28 & \\
\hline
\end{tabular}

BPTF, bromodomain PHD-finger transcription factor; KPS, Karnofsky performance score. Bold indicates a statistically significant result.

BPTF expression was significantly correlated with tumor size $(\mathrm{P}<0.001)$ and $\mathrm{WHO}$ grade $(\mathrm{P}=0.001)$, but had no statistical significance with sex, age, tumor location, tumor cystic change, tumor necrosis and KPS score (Table I). Subsequently, the survival curve was constructed and the survival rate difference between the groups was analyzed by log-rank test. The results revealed that the BPTF low-expression group had favorable overall survival (OS) $(\mathrm{P}<0.001$; Fig. 2B) and progression-free survival (PFS) $(\mathrm{P}<0.001$; Fig. $2 \mathrm{C})$ compared with the BPTF high-expression group. Furthermore, univariate and multivariate Cox regression analyses both indicated that BPTF expression was an independent prognostic factor for the OS and PFS of glioma patients (Tables II and III). These results indicated that BPTF may be a useful prognostic biomarker for glioma patients and may be involved in the progression of glioma.

BPTF enhances proliferation and invasion of human glioma cells in vitro. To study the biological function of BPTF in glioma, we constructed BPTF overexpressed (named U251 ${ }^{\text {BPTF-OE }}$ ) and knocked down (named U251 ${ }^{\text {BPTF-KD }}$ ) plasmids and transfected them into glioma U251 cells. We first examined the proliferation ability by colony-forming assay. The results revealed that BPTF overexpression increased the size and number of U251 cell colonies, while BPTF knockdown decreased the size and number of colonies compared to their control (Fig. 3A). Then, we detected the effects of BPTF on motility and migration by wound healing and Transwell assays. In comparison with the control cells, wound healing and Transwell assays revealed that BPTF overexpression significantly enhanced the motility and migration of U251 cells (Fig. 3B and C). Then, the Transwell Matrigel invasion assay revealed that BPTF overexpression increased the number of cells that invaded through the Matrigel membrane which indicated that BPTF enhanced the invasiveness ability of U251 cells (Fig. 3D). Conversely, knockdown of BPTF in U251 cells significantly reduced the motility and migration (Fig. 3B and C) and invasive capacity of cells (Fig. 3D). These results indicated that BPTF could enhance malignant characteristics of $\mathrm{U} 251$ cells in vitro. 
A

Comparision of BPTF across 25 analyses overexpression

Median rank P-value Gene

$2291.0 \quad 3.81 \mathrm{E}-4$ BPTF

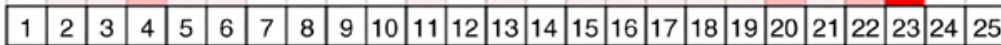

Legend

1. Anaplastic Oligoastrocytoma vs. Normal Bredel Brain 2, Cancer Res, 2005

2. Anaplastic Oligodendroglioma vs. Normal Bredel Brain 2, Cancer Res, 2005

3. Glioblastoma vs. Normal Bredel Brain 2, Cancer Res, 2005

4. Oligodendroglioma vs. Normal Bredel Brain 2, Cancer Res, 2005

5. Anaplastic Oligoastrocytoma vs. Normal French Brain, Cancer Res, 2006

6. Anaplastic Oligodendroglioma vs. Normal French Brain, Cancer Res, 2006

7. Pilocytic Astrocytoma vs. Normal Gutmann Brain, Cancer Res, 2002

8. Glioblastoma vs. Normal

Lee Brain, Cancer Cell, 2006

9. Glioblastoma vs. Normal

Liang Brain, Proc Natl Acad Sci U S A, 2005

10. Oligoastrocytoma vs. Normal

Liang Brain, Proc Natl Acad Sci U S A, 2005

11. Glioblastoma vs. Normal

Murat Brain, J Clin Oncol, 2008

12. Atypical Teratoid/Rhabdoid Tumor vs. Normal

Pomeroy Brain, Nature, 2002

13. Classic Medulloblastoma vs. Normal

Pomeroy Brain, Nature, 2002

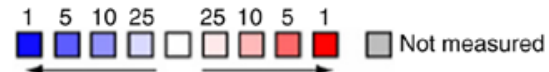

Rank for a gene is the median rank for that gene across each of the analyses.

$\mathrm{P}$-value for a gene is its P-value for the median-ranked analysis.

B

14. BPTF expression in Rickman Brain

Rickman brain statistics

Overexpression gene rank: 660 (in top 13\%)

Reporter: U05237_at v
P-value: 0.011

t- test: 3.140

Fold-change: 1.930

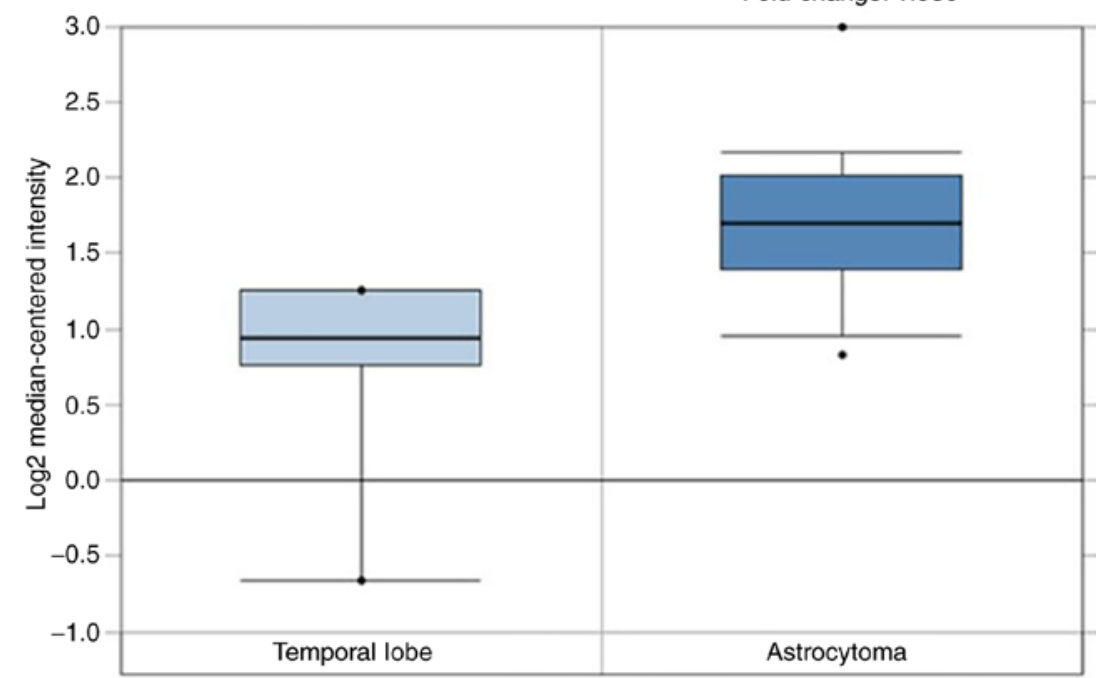

Figure 1. BPTF expression is significantly elevated in gliomas tissues. (A) The expression profile of BPTF in Oncomine ${ }^{\mathrm{TM}}$ database revealed that the expression level of BPTF in brain tumor tissue was significantly higher than that in normal brain tissue in most of the public data. The red color indicated high-rank expression, and the blue color indicated low-rank expression. (B and C) BPTF expression analysis in astrocytoma of Rickman's data (B) (P=0.011) and glioblastoma of Murat's data $(\mathrm{C})(\mathrm{P}=0.001)$ were both higher than that in normal brain tissue. 
C

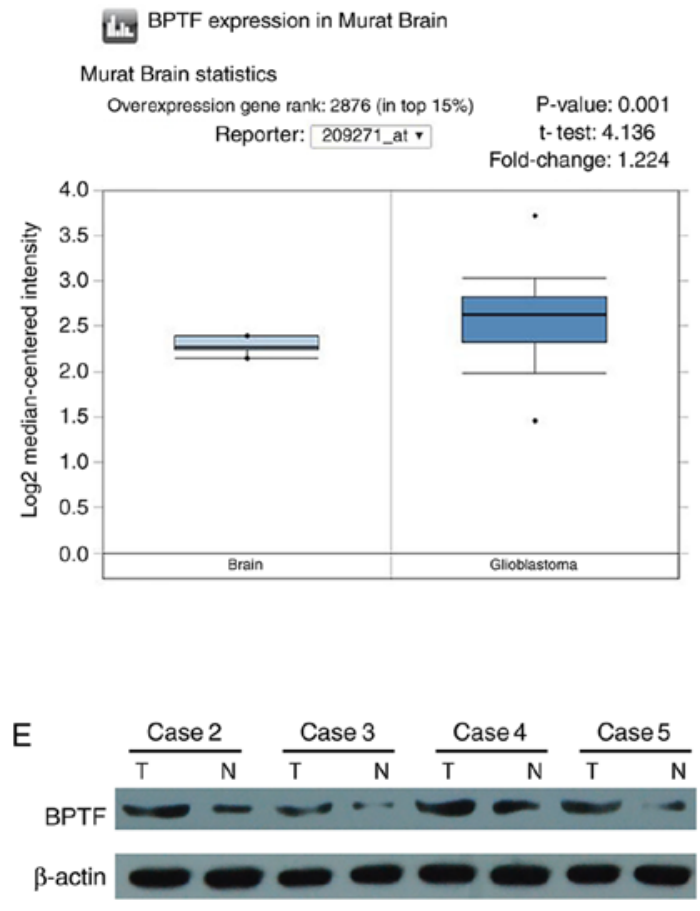

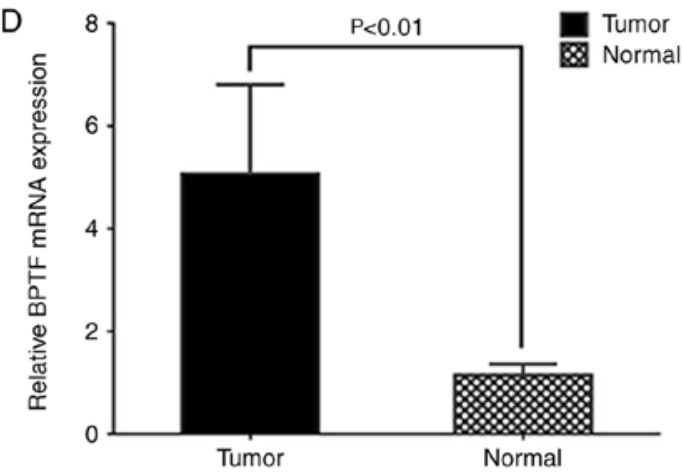

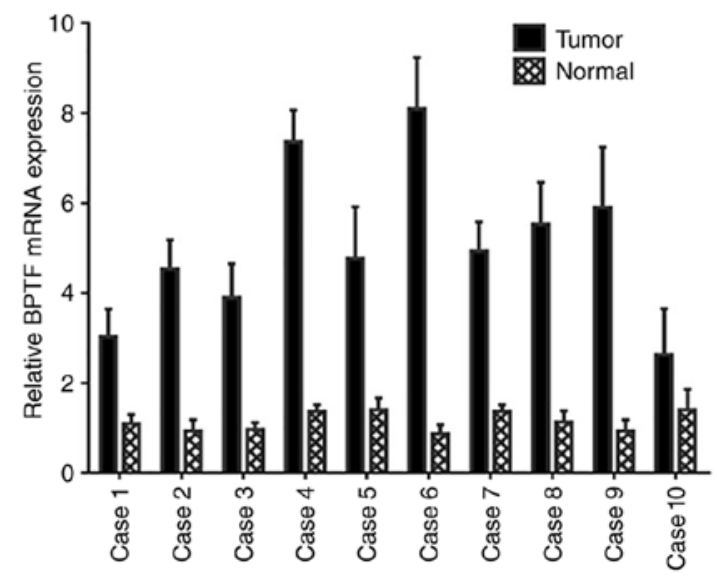

Figure 1. Continued. (B and C) BPTF expression analysis in astrocytoma of Rickman's data (B) $(\mathrm{P}=0.011)$ and glioblastoma of Murat's data (C) (P=0.001) were both higher than that in normal brain tissue. (D) Real-time PCR revealed that BPTF mRNA expression level in glioma tissues was higher than that in corresponding normal brain tissues $(\mathrm{P}<0.01)$. (E) The representative western blotting results revealed that BPTF protein expression level in glioma tissues was higher than that in corresponding normal brain tissues.
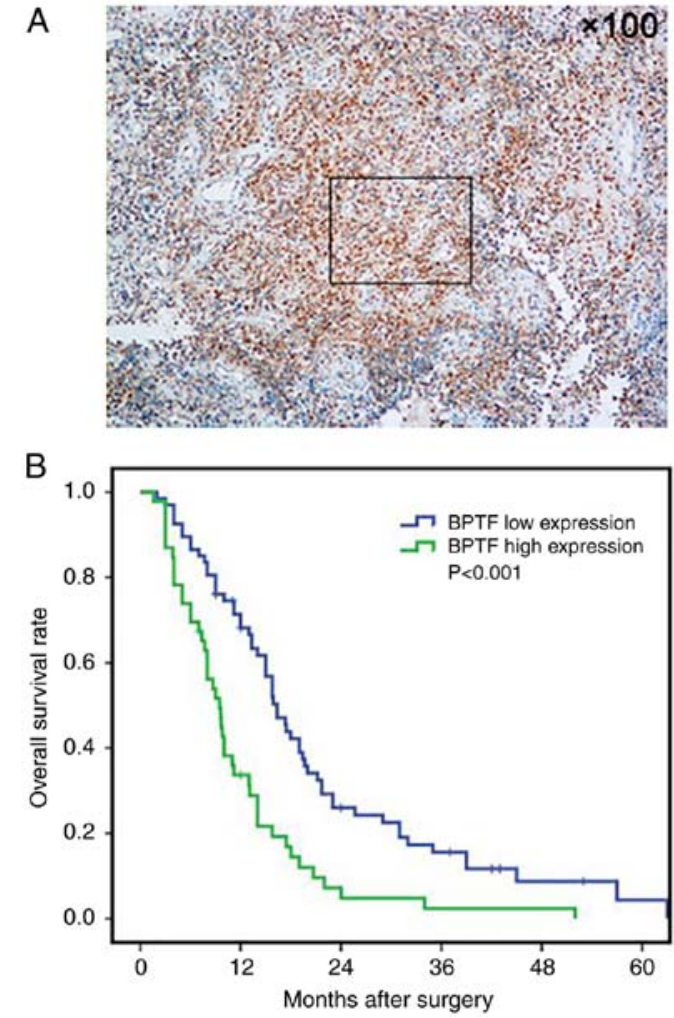
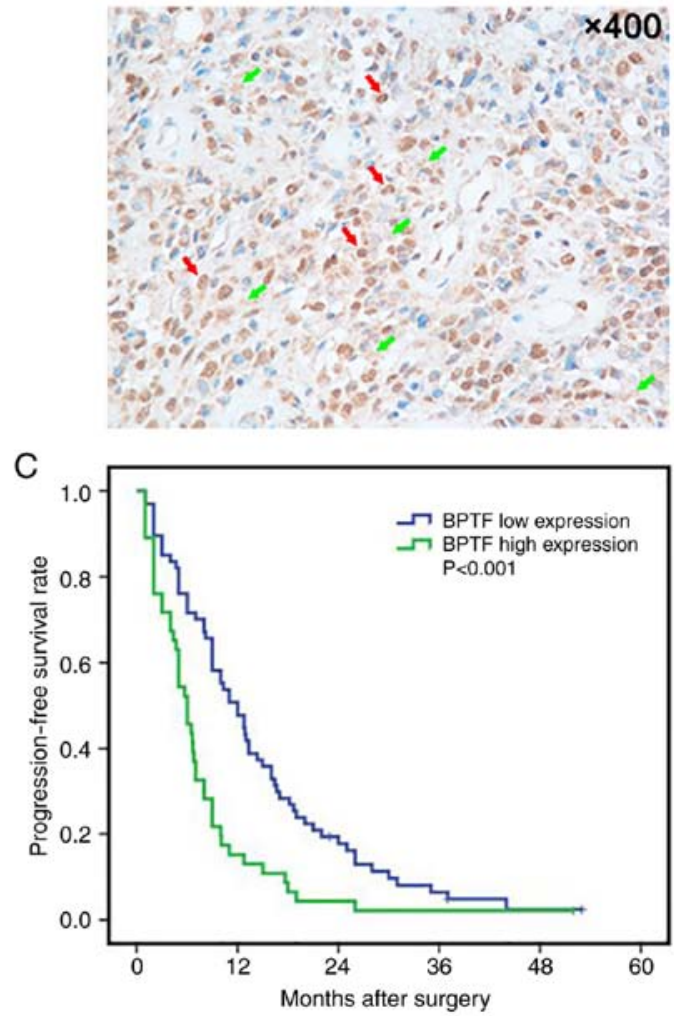

Figure 2. BPTF expression is associated with the survival of glioma patients. (A) Representative IHC images of BPTF expression in glioma tissues revealed that BPTF positive staining was mainly expressed in the nuclei (red arrows) and/or the cytoplasm (green arrows) of tumor cells. (B) The survival curve revealed that the low-BPTF expression group had favorable overall survival than that in the high-expression group ( $<<0.001)$. (C) The survival curve revealed that the low-BPTF expression group had favorable progression-free survival than that in the high-expression group $(\mathrm{P}<0.001)$. 
Table II. Univariate and multivariate analyses of factors affecting overall survival (OS) in glioma patients.

\begin{tabular}{|c|c|c|c|c|}
\hline \multirow[b]{2}{*}{ Variables } & \multicolumn{2}{|c|}{ Univariate analysis } & \multicolumn{2}{|c|}{ Multivariate analysis } \\
\hline & $\mathrm{HR}(95 \% \mathrm{CI})$ & $\mathrm{P}$-value & $\mathrm{HR}(95 \% \mathrm{CI})$ & P-value \\
\hline Sex & $0.897(0.605-1.331)$ & 0.591 & & \\
\hline Age (years) & $1.111(0.749-1.648)$ & 0.603 & & \\
\hline Location & $1.062(0.926-1.217)$ & 0.389 & & \\
\hline Tumor size & $2.270(1.514-3.405)$ & $<0.001$ & $1.683(1.070-2.648)$ & 0.024 \\
\hline Cystic change & $0.885(0.574-1.364)$ & 0.579 & & \\
\hline Necrosis & $1.276(0.823-1.978)$ & 0.277 & & \\
\hline WHO grade & $3.261(2.121-5.014)$ & $<0.001$ & $2.824(1.776-4.489)$ & $<0.001$ \\
\hline KPS score & $0.848(0.572-1.257)$ & 0.411 & & \\
\hline BPTF expression & $2.259(1.506-3.387)$ & $<0.001$ & $1.789(1.125-2.846)$ & 0.014 \\
\hline
\end{tabular}

HR, hazard ratio; CI, confidence interval; BPTF, bromodomain PHD-finger transcription factor; KPS, Karnofsky performance score. Bold indicates a statistically significant result.

Table III. Univariate and multivariate analyses of factors affecting progression-free survival (PFS) in glioma patients.

\begin{tabular}{|c|c|c|c|c|}
\hline \multirow[b]{2}{*}{ Variables } & \multicolumn{2}{|c|}{ Univariate analysis } & \multicolumn{2}{|c|}{ Multivariate analysis } \\
\hline & $\mathrm{HR}(95 \% \mathrm{CI})$ & P-value & $\mathrm{HR}(95 \% \mathrm{CI})$ & P-value \\
\hline Sex & $0.927(0.635-1.355)$ & 0.696 & & \\
\hline Age (years) & $1.122(0.766-1.644)$ & 0.554 & & \\
\hline Location & $1.089(0.956-1.240)$ & 0.200 & & \\
\hline Tumor size & $2.127(1.441-3.140)$ & $<0.001$ & $1.658(1.076-2.554)$ & 0.022 \\
\hline Cystic change & $0.928(0.618-1.395)$ & 0.720 & & \\
\hline Necrosis & $1.226(0.800-1.877)$ & 0.349 & & \\
\hline WHO grade & $2.611(1.725-3.952)$ & $<0.001$ & $2.118(1.354-3.312)$ & 0.001 \\
\hline KPS score & $0.922(0.630-1.348)$ & 0.674 & & \\
\hline BPTF expression & $2.023(1.366-2.995)$ & $<0.001$ & $1.603(1.020-2.521)$ & 0.041 \\
\hline
\end{tabular}

HR, hazard ratio; CI, confidence interval. Bold indicates a statistically significant result.

BPTF promotes glioma growth and invasion via Myc signaling. Next, we wanted to explore the potential molecular mechanism of BPTF in the promotion of glioma proliferation and invasion. We first utilized the Gene-Cloud of Biotechnology (GCBI; https://www.gcbi.com.cn/gclib/html/index) database to search for potential interacting genes and found that Myc was evidently a potential candidate (Fig. 4A). Since Myc signaling is vital for proliferation and invasion in various tumors, we further searched for the association of BPTF and Myc in COXPRESdb and Oncomine databases. The results revealed that BPTF was co-expressed with MYCBP2 (Fig. 4B and C) and MYCBPAP (Fig. 4D), both of which are Myc-binding association proteins. According to these bioinformatics results, we further detected c-Myc expression, which is the core member of Myc signaling, in BPTF overexpressed or knocked down $\mathrm{U} 251$ cells. The results revealed that c-Myc mRNA and protein expression level were significantly increased in U251 ${ }^{\text {BPTF-OE }}$ cells than the control cells but decreased in U251 ${ }^{\text {BPTF-KD }}$ cells (Fig. 4E and F). These data indicated that BPTF promoted glioma proliferation and invasion via Myc signaling.

\section{Discussion}

Glioma can occur at any age, regardless of sex or ethnicity. On the basis of their histopathology, gliomas are divided into grades I-IV according to the World Health Organisation (WHO) grading system $(3,16,17)$. Despite advances in surgery, radiotherapy and chemotherapy that have been achieved, current therapies against gliomas are still not effective enough and with poor long-term survival. Grades III and IV gliomas are more aggressive and difficult to treat owing to frequent dysfunction of tumor suppressors and oncogenes (18). Therefore, the present study explored the role of BPTF as an oncogene in glioma and provided a potential option for prognostic prediction and targeted therapy of glioma.

The present study by combining an online bioinformatics database and clinical specimens, first revealed that BPTF expression was highly expressed in gliomas and correlated with poor survival. These findings were consistent with previous research on other tumors, such as hepatocellular carcinoma (HCC), and lung and colorectal cancer (CRC) $(13,19)$. We also explored 
A

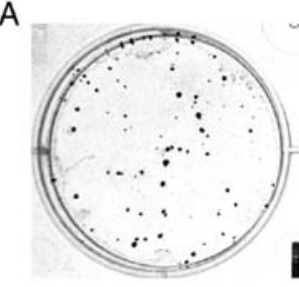

B
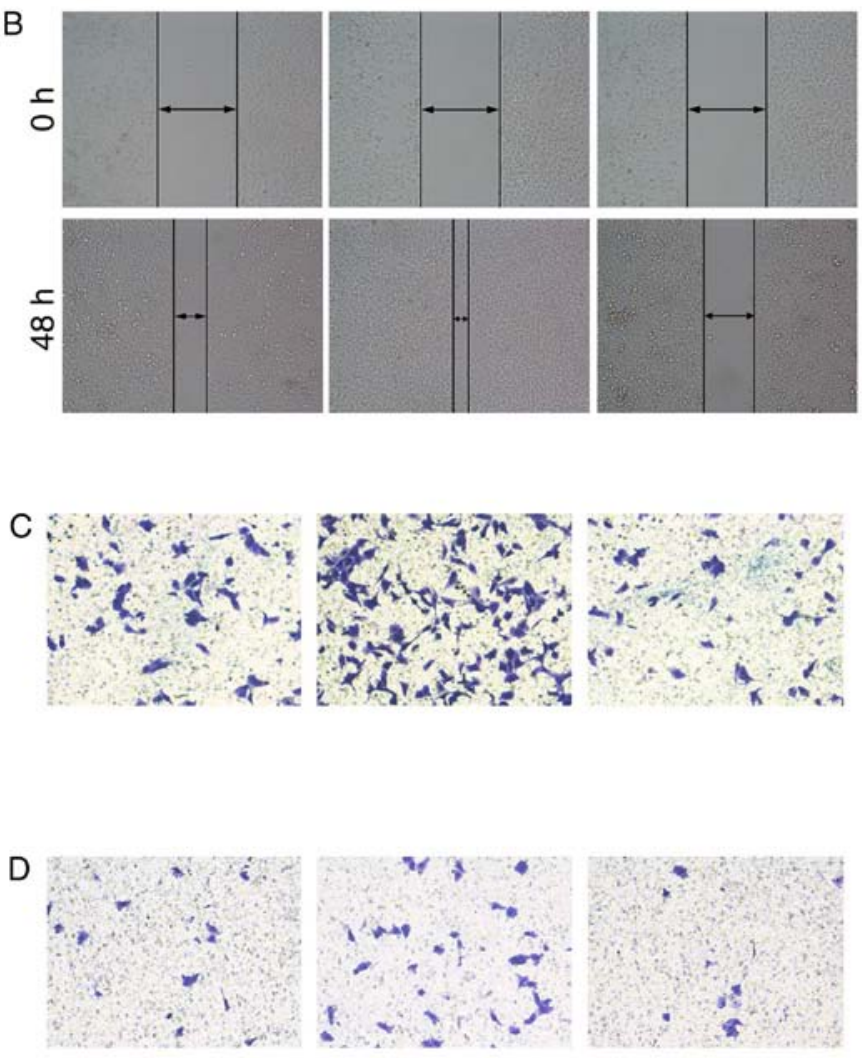
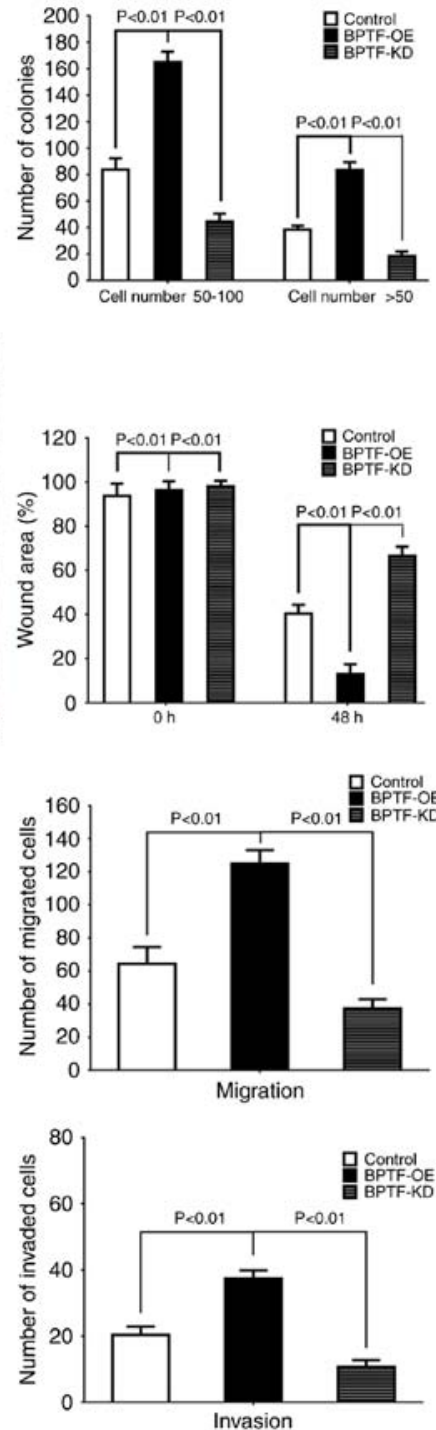

Figure 3. BPTF enhances proliferation and invasion of human glioma cells in vitro. (A) The colony-forming assay revealed that BPTF expression increased the colony size and number of $\mathrm{U} 251$ cells $(\mathrm{P}<0.01)$. (B) A wound healing assay revealed that BPTF expression increased the wound healing rate of $\mathrm{U} 251$ cells $(\mathrm{P}<0.01)$. (C) A Transwell assay revealed that BPTF expression increased the number of $\mathrm{U} 251$ cells that migrated across the chamber $(\mathrm{P}<0.01)(\mathrm{magnification}$, $\mathrm{x} 100$ ). (D) A Transwell Matrigel invasion assay revealed that BPTF expression increased the number of U251 cells that invaded across the Matrigel membrane $(\mathrm{P}<0.01)$ (magnification, $\mathrm{x} 100)$.
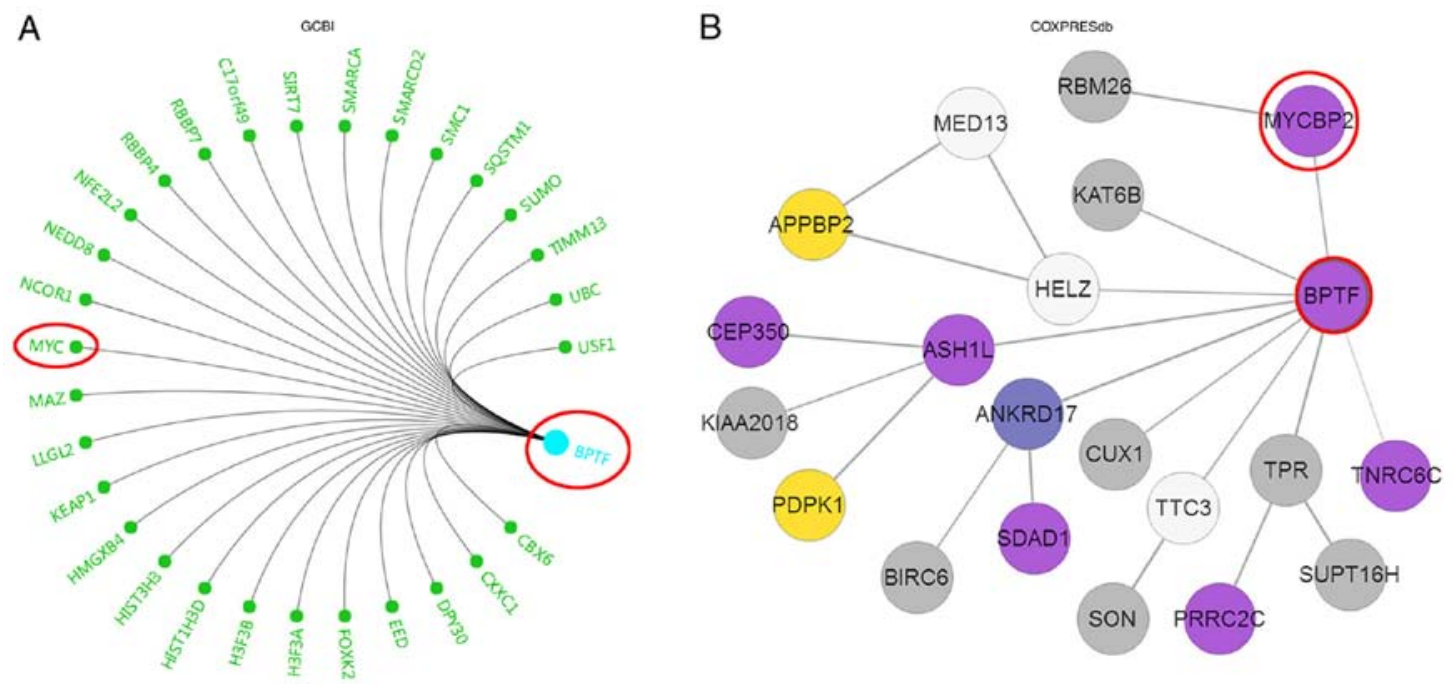

Figure 4. BPTF promotes glioma growth and invasion via Myc signaling. (A) The Gene-Cloud of Biotechnology (GCBI) database analysis revealed that Myc interacted with BPTF. (B) The COXPRESdb database analysis revealed that MYCBP2 was predicted to interact with BPTF. 
C

COXPRESdb

\begin{tabular}{|c|c|l|c|c|}
\hline Species & Symbol & Function $^{*}$ & Entrez gene ID* & Selected probe \\
\hline Hsa & BPTF & Bromodomain PHD finger transcription factor & 2186 & 207186_s_at \\
\hline Hsa & MYCBP2 & MYC binding protein 2, E3 ubiquitin protein ligase & 23077 & 201959_s_at \\
\hline
\end{tabular}

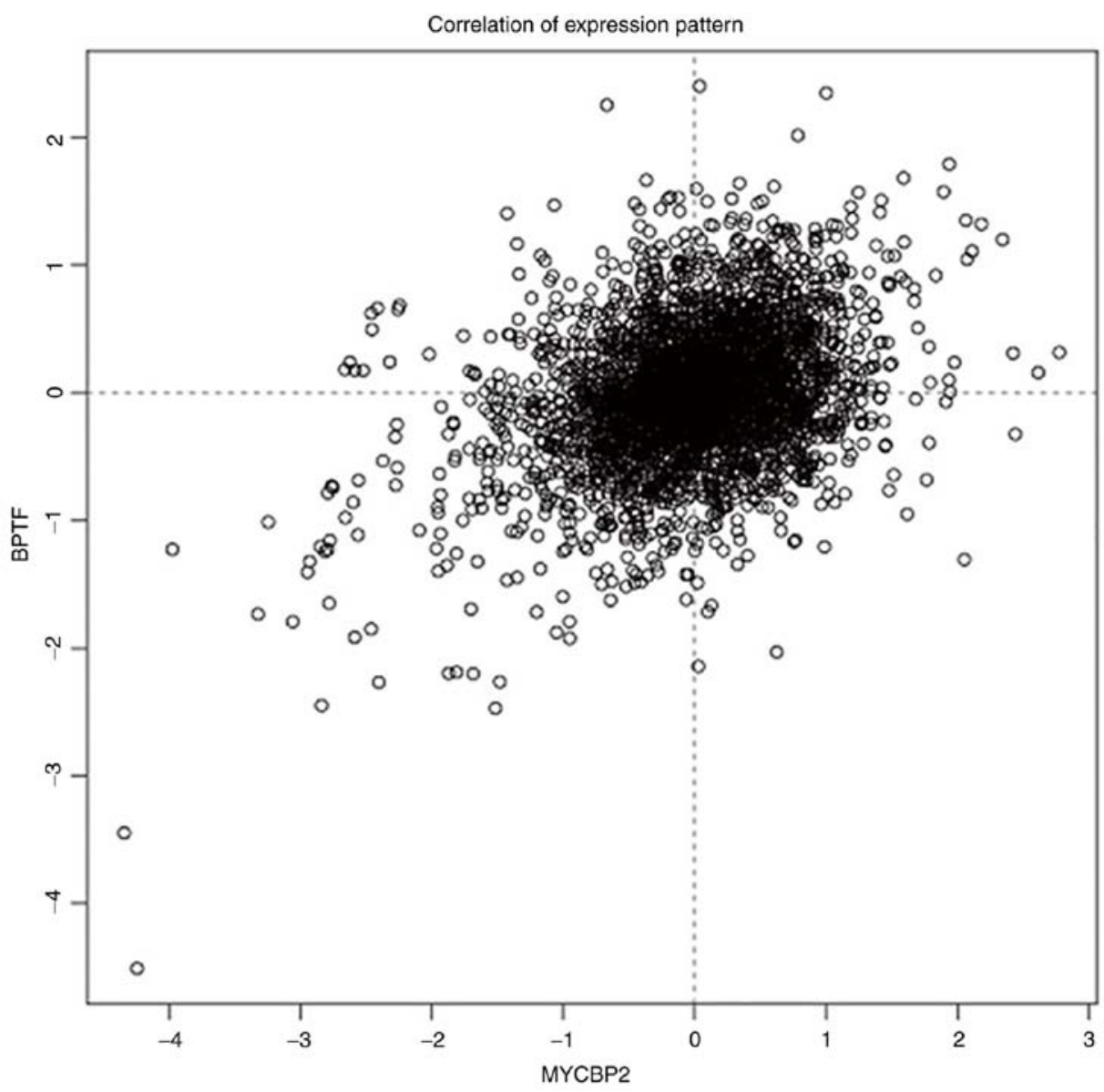

Figure 4. Continued. (C) The COXPRESdb database co-expression analysis revealed that BPTF and MYCBP2 expression had a positive linear correlation.

the clinical significance of BPTF in glioma patients and found that the expression of BPTF in glioma tissues was correlated with histopathological grade and tumor size, indicating that BPTF expression may be related with glioma progression. The survival curves and univariate and multivariate analyses for survival also revealed that high BPTF expression was an independent risk factor affecting the prognosis of glioma patients, indicating that the elevated expression of BPTF could be a useful predictor for predicting prognosis of glioma patients. The clinicopathological and prognostic findings were also consistent with the researches in melanoma, HCC and CRC $(12,19,20)$. All this evidence indicated that BPTF may be a pan-oncogene in tumors, and extended the knowledge of the biological role of BPTF, especially in the field of tumor research.

Notably, we also investigated the functional role of BPTF in glioma. Our in vitro experiments indicated that BPTF could increase the proliferation and invasiveness of glioma cells. BPTF was first identified and characterized in 2000 and was considered to play a role in hormonally-regulated, chromatin-mediated regulation of transcription during proliferation (8). Then, further studies found that BPTF played an important role in embryo development, stemness maintenance and self-renewal capacity of stem/progenitor cells (21-23). These known biological functions were revealed to be correlated with tumor progression. Research also found that BPTF was overexpressed in many cancer cell lines and had the ability to promote cancer cell growth (24). In addition, further studies revealed the correlation of BPTF aberrant expression with cancer and found that BPTF could promote tumor cell proliferation, invasion and metastasis, thus resulting in poor prognosis $(13,25-27)$. The present study also provided first-hand data of the promoting effect of BPTF on proliferation and invasion in glioma cells, which was consistent with studies in other types of cancers (24-27) and not reported in glioma before.

The present study also explored the potential molecular mechanism of BPTF in glioma. The bioinformatics and online public databases provided a preliminary correlation of BPTF with Myc signaling. Since the study conducted in 2000 first found that BPTF could interact with the Myc-associated zinc finger protein, studies confirmed that BPTF was a crucial c-Myc co-factor and played a key role in c-Myc-mediated tumor progression. The present study also revealed the correlation and potential interaction of BPTF and c-Myc in glioma $(11,26,28)$. These data further indicated that BPTF may be a useful therapeutic target for c-Myc aberrantly-expressed tumors. The main 
D Oncomine

Genes coamplified with BPTF in Beroukhim Multi-cancer Grouped by Brain and CNS Cancer type (log2 copy number units)
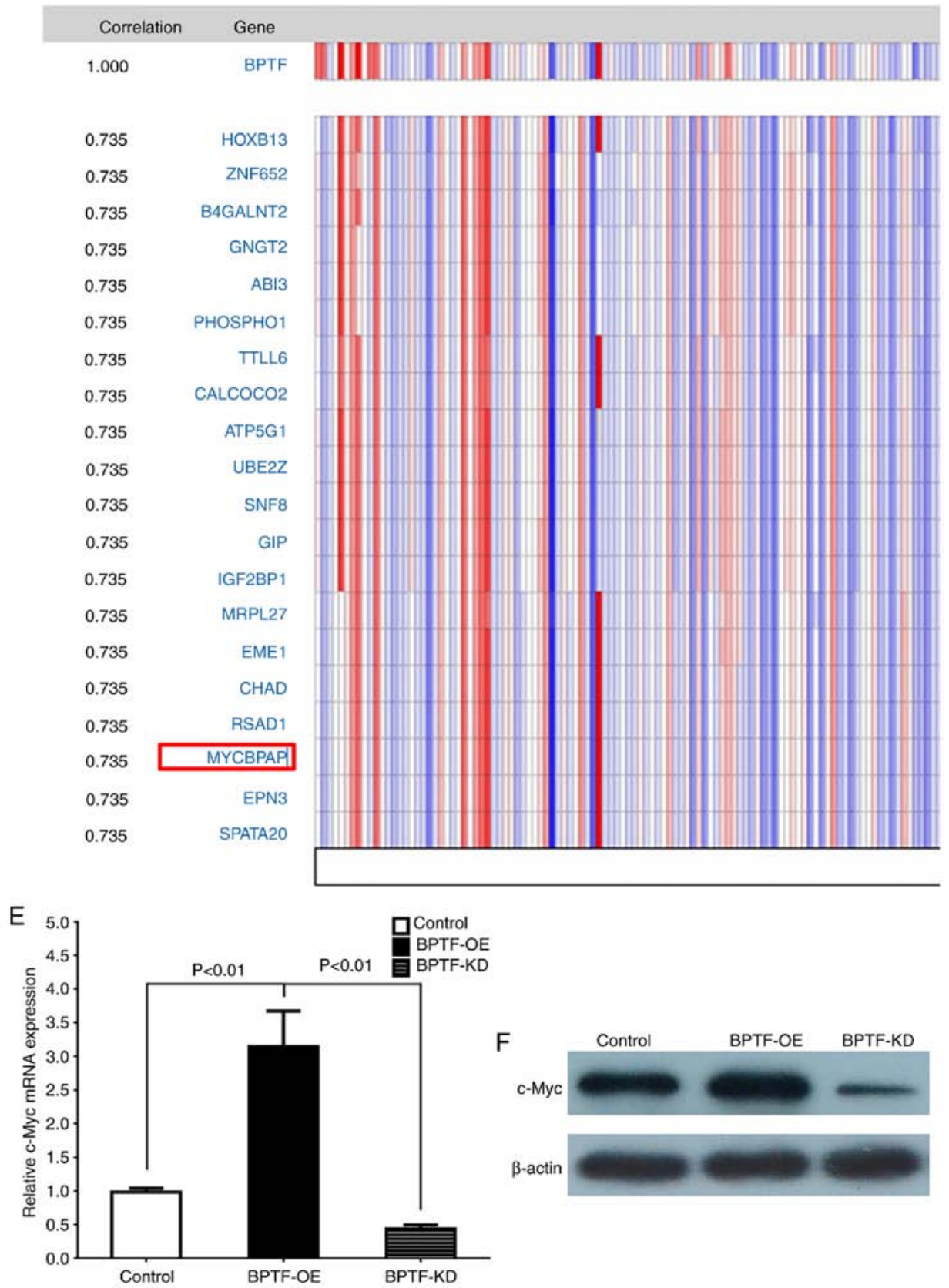

Figure 4. Continued. (D) The Oncomine database analysis also revealed that BPTF and MYCBPAP expression had a positive correlation. (E) Real-time PCR revealed that c-Myc mRNA expression was upregulated in U251 ${ }^{\mathrm{BPTF}-\mathrm{OE}}$ cells, but downregulated in U251 ${ }^{\mathrm{BPTF}-\mathrm{KD}}$ cells. (F) Western blotting revealed that c-Myc protein expression was upregulated in $\mathrm{U} 251^{\mathrm{BPTF}-\mathrm{OE}}$ cells, but downregulated in U251 ${ }^{\mathrm{BPTF}-\mathrm{KD}}$ cells.

shortcomings of our study are as follows: i) we did not conduct gain- and loss-of-function analyses for c-Myc with BPTF interference; ii) this study did not provide detailed molecular mechanism analysis of how BPTF regulated c-Myc in glioma; iii) we did not provide in vivo intervention experiments examining the therapeutic effect of BPTF inhibition. Therefore, our future research directions will be aimed at these shortcomings.

In summary, our results revealed that BPTF was overexpressed in glioma and could promote proliferation and invasion of glioma cells via Myc signaling. Moreover, BPTF may be a valuable prognostic marker and therapeutic target for glioma patients.

\section{Acknowledgements}

Not applicable.

\section{Funding}

No funding was received. 


\section{Availability of data and materials}

The datasets used during the present study are available from the corresponding author upon reasonable request.

\section{Authors' contributions}

YP, FY, ZL and LC conceived the study and wrote the manuscript; YP, FY, YL, GW and ZL conducted the experiments and contributed to the analysis of data. YP, FY, YL and GW collected the clinical samples and the corresponding clinical data. YP, FY and LC revised the manuscript. All authors read and approved the manuscript and agree to be accountable for all aspects of the research in ensuring that the accuracy or integrity of any part of the work are appropriately investigated and resolved.

\section{Ethics approval and consent to participate}

The study was approved by the Ethics Committee of Haikou People's Hospital in accordance with the Declaration of Helsinki. All patients provided signed written informed consent.

\section{Patient consent for publication}

Not applicable.

\section{Competing interests}

The authors declare that they have no competing interests.

\section{References}

1. Kohler BA, Ward E, McCarthy BJ, Schymura MJ, Ries LA, Eheman C, Jemal A, Anderson RN, Ajani UA and Edwards BK: Annual report to the nation on the status of cancer, 1975-2007, featuring tumors of the brain and other nervous system. J Natl Cancer Inst 103: 714-736, 2011.

2. Popova SN, Bergqvist M, Dimberg A, Edqvist PH, Ekman S, Hesselager G, Ponten F, Smits A, Sooman L and Alafuzoff I: Subtyping of gliomas of various WHO grades by the application of immunohistochemistry. Histopathology 64: 365-379, 2014.

3. Ho VK, Reijneveld JC, Enting RH, Bienfait HP, Robe P, Baumert BG, Visser O and Dutch Society for Neuro-Oncology (LWNO): Changing incidence and improved survival of gliomas. Eur J Cancer 50: 2309-2318, 2014.

4. Hanahan D and Robert W: Hallmarks of cancer: The next generation. Cell 144: 646-674, 2011.

5. Stine ZE, Walton ZE, Altman BJ, Hsieh AL and Dang CV: MYC, metabolism, and cancer. Cancer Discov 5: 1024-1039, 2015.

6. Principe DR, Doll JA, Bauer J, Jung B, Munshi HG, Bartholin L, Pasche B, Lee C and Grippo PJ: TGF- $\beta$ : Duality of function between tumor prevention and carcinogenesis. J Natil Cancer Inst 106: djt369, 2014.

7. Wysocka J, Swigut T, Xiao H, Milne TA, Kwon SY, Landry J, Kauer M, Tackett AJ, Chait BT, Badenhorst P, et al: A PHD finger of NURF couples histone $\mathrm{H} 3$ lysine 4 trimethylation with chromatin remodelling. Nature 442: 86-90, 2006.

8. Jones MH, Hamana $\mathrm{N}$ and Shimane M: Identification and characterization of BPTF, a novel bromodomain transcription factor. Genomics 63: 35-39, 2000.

9. Goller T, Vauti F, Ramasamy S and Arnold HH: Transcriptional regulator $\mathrm{BPTF} / \mathrm{FAC} 1$ is essential for trophoblast differentiation during early mouse development. Mol Cell Biol 28: 6819-6827, 2008 .

10. Ma Y, Liu X, Liu Z, Wei S, Shang H, Xue Y, Cao Y, Meng A and Wang Q: The chromatin remodeling protein Bptf promotes posterior neuroectodermal fate by enhancing Smad2-activated wnt8a expression. J Neurosci 35: 8493-8506, 2015.
11. Richart L, Real FX and Sanchez-Arevalo Lobo VJ: c-MYC partners with BPTF in human cancer. Mol Cell Oncol 3: e1152346, 2016.

12. Xiao S, Liu L, Lu X, Long J, Zhou X and Fang M: The prognostic significance of bromodomain PHD-finger transcription factor in colorectal carcinoma and association with vimentin and E-cadherin. J Cancer Res Clin Oncol 141: 1465-1474, 2015.

13. Dai M, Lu JJ, Guo W, Yu W, Wang Q, Tang R, Tang Z, Xiao Y, $\mathrm{Li} \mathrm{Z}$, Sun W, et al: BPTF promotes tumor growth and predicts poor prognosis in lung adenocarcinomas. Oncotarget 6 : 33878-33892, 2015.

14. Livak KJ and Schmittgen TD: Analysis of relative gene expression data using real-time quantitative PCR and the $2-\Delta \Delta C$ T method. Methods 25: 402-408, 2001.

15. Mayes K, Elsayed Z, Alhazmi A, Waters M, Alkhatib SG, Roberts M, Song C, Peterson K, Chan V, Ailaney N, et al: BPTF inhibits NK cell activity and the abundance of natural cytotoxicity receptor co-ligands. Oncotarget 8: 64344-64357, 2017.

16. Boruah D, Deb P, Srinivas V and Mani NS: Morphometric study of nuclei and microvessels in gliomas and its correlation with grades. Microvasc Res 93: 52-61, 2014.

17. Louis DN, Perry A, Reifenberger G, von Deimling A, Figarella-Branger D, Cavenee WK, Ohgaki H, Wiestler OD, Kleihues P and Ellison DW: The 2016 world health organization classification of tumors of the central nervous system: A summary. Acta Neuropathol 131: 803-820, 2016.

18. Minniti G, Muni R, Lanzetta G, Marchetti P and Enrici RM: Chemotherapy for glioblastoma: Current treatment and future perspectives for cytotoxic and targeted agents. Anticancer Res 29: 5171-5184, 2009.

19. Xiao S, Liu L, Fang M, Zhou X, Peng X, Long J and Lu X: BPTF associated with EMT indicates negative prognosis in patients with hepatocellular carcinoma. Dig Dis Sci 60: 910-918, 2015.

20. Dar AA, Majid S, Bezrookove V, Phan B, Ursu S, Nosrati M, De Semir D, Sagebiel RW, Miller JR III, Debs R, et al: BPTF transduces MITF-driven prosurvival signals in melanoma cells. Proc Natl Acad Sci USA 113: 6254-6258, 2016.

21. Landry J, Sharov AA, Piao Y, Sharova LV, Xiao H, Southon E, Matta J, Tessarollo L, Zhang YE, Ko MS, et al: Essential role of chromatin remodeling protein Bptf in early mouse embryos and embryonic stem cells. PLoS Genet 4: e1000241, 2008.

22. Xu B, Cai L, Butler JM, Chen D, Lu X, Allison DF, Lu R, Rafii S, Parker JS, Zheng D and Wang GG: The chromatin remodeler BPTF activates a stemness gene-expression program essential for the maintenance of adult hematopoietic stem cells. Stem Cell Reports 10: 675-683, 2018.

23. Frey WD, Chaudhry A, Slepicka PF, Ouellette AM, Kirberger SE, Pomerantz WCK, Hannon GJ and Dos Santos CO: BPTF maintains chromatin accessibility and the self-renewal capacity of mammary gland stem cells. Stem Cell Reports 9: 23-31, 2017.

24. Buganim Y, Goldstein I, Lipson D, Milyavsky M, Polak-Charcon S, Mardoukh C, Solomon H, Kalo E, Madar S, Brosh R, et al: A novel translocation breakpoint within the BPTF gene is associated with a pre-malignant phenotype. PLoS One 5: e9657, 2010.

25. Dar AA, Nosrati M, Bezrookove V, de Semir D, Majid S, Thummala S, Sun V, Tong S, Leong SP, Minor D, et al: The role of BPTF in melanoma progression and in response to BRAF-targeted therapy. J Natl Cancer Inst 107: djv034, 2015.

26. Richart L, Carrillo-de Santa Pau E, Río-Machín A, de Andrés MP, Cigudosa JC, Lobo VJ and Real FX: BPTF is required for c-MYC transcriptional activity and in vivo tumorigenesis. Nat Commun 7: 10153, 2016.

27. Li Y, Li J, Luo M, Zhou C, Shi X, Yang W, Lu Z, Chen Z, Sun N and He J: Novel long noncoding RNA NMR promotes tumor progression via NSUN2 and BPTF in esophageal squamous cell carcinoma. Cancer Lett 430: 57-66, 2018.

28. Jordan-Sciutto KL, Dragich JM, Caltagarone J, Hall DJ and Bowser R: Fetal Alz-50 clone 1 (FAC1) protein interacts with the Myc-associated zinc finger protein (ZF87/MAZ) and alters its transcriptional activity. Biochemistry 39: 3206-3215, 2000 . International (CC BY-NC-ND 4.0) License. 